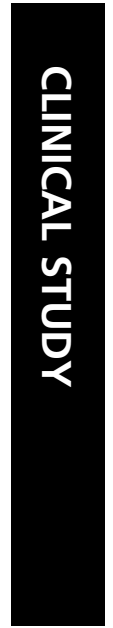

\section{UBM-guided chamber angle surgery for glaucoma management: an experimental study}

\author{
Abstract \\ Purpose The aim of this experimental study \\ was to investigate the potential of ultrasound \\ bimicroscopy (UBM)-guided chamber angle \\ surgery as an alternative or supplement to \\ gonioscopy and intraocular microendoscopy \\ for intraoperative control. \\ Methods In 15 porcine cadaver eyes, \\ mechanical goniopuncture or punctual Er:YAG \\ laser trabecular ablation was performed \\ without operating microscope or gonioscopy, \\ but with real-life ultrasound biomicroscopy \\ monitoring with a $50 \mathrm{MHz}$ transducer. \\ Intraoperative localization of the \\ microsurgical instruments and tissue- \\ instrument contact were qualitatively \\ evaluated.
}

Results The instruments could be clearly visualized within the chamber angle and disturbing artefacts were only minimal when using mechanically fixed instruments in slow motion. Topographic localization, tissue contact, and penetration depth of the instruments entering the scleral were well illustrated as far as the technical

Department of Ophthalmology University of Cologne Germany

Correspondence:

TS Dietlein

Department of

Ophthalmology University of Cologne Joseph-Stelzmann-Str. 9 50931 Köln, Germany Tel: +492214784300 Fax: +492214784347

E-mail: aia47@ uni-koeln.de

Received: 13 August 2001 Accepted in revised form: 11 June 2002 resolution limits of UBM would

allow.

Conclusions UBM can be used intraoperatively to monitor the correct manoeuvring of microsurgical instruments in selected $a b$ interno procedures. Some adapations and further modifications of the technique presented here will be necessary before UBM-guided surgery can be considered for clinical use in humans.

Eye (2003) 17, 340-345. doi:10.1038/

sj.eye. 6700328

Keywords: ultrasound biomicroscopy; glaucoma surgery; laser trabecular ablation; goniopuncture; gonioscopy
TS Dietlein, BF Engels, PC Jacobi and GK Krieglstein

Introduction

Good visualization of microsurgical procedures in ophthalmology is the key to their success. In cataract and glaucoma surgery, adequate surveillance of surgical steps is guaranteed by magnification through the operating microscope, but depends on the clarity of the cornea. In antiglaucomatous chamber angle surgery such as goniotomy, ${ }^{1-3}$ clear viewing of the chamber angle structures can be critically reduced by the quality of the gonioscope, the clarity of the cornea, or intraoperative reflux bleeding from Schlemm's canal. The identification of chamber angle details is essential for most of the $a b$ interno interventions targeting the trabecular meshwork, for example goniotomy, goniopuncture, laser trabeculopuncture, or trabecular ablation. ${ }^{4-7}$ Microendoscopically guided trabecular surgery may be a promising alternative to gonioscopy in cases of corneal opacity. ${ }^{8,9}$ However, neither gonioscopy nor microendoscopy is able to provide valuable intraoperative information about the tissue depth that the surgeon's instruments have reached or about the tissue density beyond the trabecular meshwork.

This is an area where ultrasound biomicroscopy (UBM) may be useful. ${ }^{10,11}$ However, several factors could limit the intraoperative use of UBM for chamber angle surgery. ${ }^{12,13}$ Firstly, the monitor field represents a two-dimensional tissue section of only $5 \times 5 \mathrm{~mm}$ and, secondly, surgical instruments made of stainless steel or similar echogenic material form a strongly echo-dense foreign body causing massive reflection of ultrasound waves. The aim of our study was to investigate whether UBM guidance of a chamber angle procedure is feasible at all without the aid of an operating microscope, gonioscopy, or microendoscopy. 


\section{Methods}

A total of 15 freshly enucleated porcine cadaver eyes, maintained for less than $5 \mathrm{~h}$ in balanced salt solution (BSS) at $4^{\circ} \mathrm{C}$, were taken from the local slaughterhouse. Eyes were fixed in a special eye-holder for anterior segment surgery. After performing a 1-mm cornea incision at the limbus, the anterior chamber was filled with viscoelastics (eg Healon GV). Surgical instruments were introduced into the anterior chamber via this paracentesis and moved in towards the contralateral chamber angle. Further axial movements of the instruments were then performed with the help of a special mechanical supporter, thus enabling highly controlled and slow intraocular manoeuvres. Ultrasound biomicroscopy was performed with a high-frequencey (50 MHz) transducer (Model 840, Zeiss-Humphrey, Hymphrey-Instruments, Munich, Germany). The scanning probe is suspended from and can be fixed by an articulated arm to decrease motion artefacts. As a coupling medium, we used a high-viscosity gel (eg Marquette Hellige Medical Systems, Germany) without an eye cup. As goniopuncture and laser trabecular ablation are surgical procedures requiring movements solely along the meridional axis, UBM examination included mainly meridional slices to supervise the surgical procedure. Lateral motions of the instruments were not monitored by UBM, because only one surgical manoeuvre was performed per eye. For goniopuncture, the needle approached the trabecular tissue and was slowly forced through the tissue until the tip had penetrated half-way through the sclera. For laser trabecular ablation, the tip penetrated the tissue to a similar distance and the endoprobe-tissue contact was carefully monitored. In order to study the potential of UBM for intraoperative evaluation of laser ablation, applied total energy was varied as well as the localization of the laser ablation zones (trabecular meshwork and peripheral cornea). For $a b$ interno laser ablation, an ER:YAG laser $(2.94 \mu \mathrm{m}$, Aesculap Meditec, MCL 29) was used. The pulse length was $200 \mu \mathrm{s}$, and single pulse energy was varied between 6 and $12 \mathrm{~mJ}$ at 1-4 Hz. Laser energy was delivered via an articulated zirconium fluoride optical fibre and a quartz fibre endoprobe with $320 \mu \mathrm{m}$ core diameter (coating diameter, $385 \mu \mathrm{m}$; surrounding metal tube, $850 \mu \mathrm{m}$ ) (Figure 1). For goniopuncture, a stainless-steel 31-gauge needle with an outer diameter of $260 \mu \mathrm{m}$ (Hamilton Bonaduz, Switzerland) was used, articulated to a $2 \mathrm{ml}$ syringe (Figure 1). After the procedure, anterior eye cups were fixed in a 5\% formalin solution and processed for qualitative topographic histological analysis. After tissue dehydrating, embedding, sectioning ( $5 \mu \mathrm{m}$ serial sections), and staining (haematoxylin-eosin), light

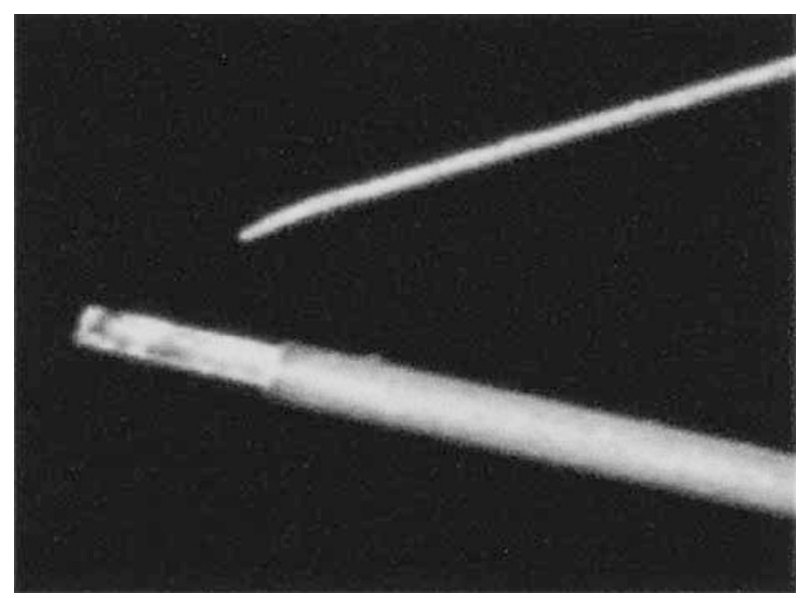

Figure 1 Above: Stainless-steel 33-gauge needle (Hamilton Bonaduz AG, Switzerland) used for mechanical goniopuncture. Below: $320 \mu \mathrm{m}$ (core diameter) laser endoprobe (Aesculap Meditec, Germany) used for ER:YAG laser ablation.

microscopy was performed in order to verify topographic localization of the surgical effects. To avoid confusion over the topographic interpretation of histologic analysis, we performed only one surgical procedure per eye. Using a microscopic grid, quantitative measurements of the crater depth, inner crater diameter and the distance between the crater and the iris root were undertaken in these eyes in which laser craters of more than $200 \mu \mathrm{m}$ depth were created.

\section{Results}

Visualization of the surgical instruments in the peripheral chamber angle was excellent, although the process of finding the best meridional section illustration was relatively time consuming ranging between 1 and $5 \mathrm{~min}$. Ultrasound artefacts were always present distal to the instruments, but did not disturb topographic orientation during the procedure. Artefacts caused by the smaller instrument were naturally less marked than those from the larger laser endoprobe (Figures 2 and 3), and by careful changing of the ultrasound probe lateral to the instrument axis, the artefacts could be reduced. UBM guidance allowed identification of the typical instrument silhouette (Figures 4 and 5) and a detailed evaluation of the instrument-tissue contact area (Figure 4). The surgeon's applanation force along the axis of the instruments could be kinetically visualized by the motion and distortion of the tissue surrounding the instrument tip. The tip of the instrument could be visualized clearly even after having entered the sclera, thus enabling the surgeon to evaluate the depth of penetration of the instruments. Owing to the limited tissue effects of single laser pulses and to emission-related artefacts such as gas 


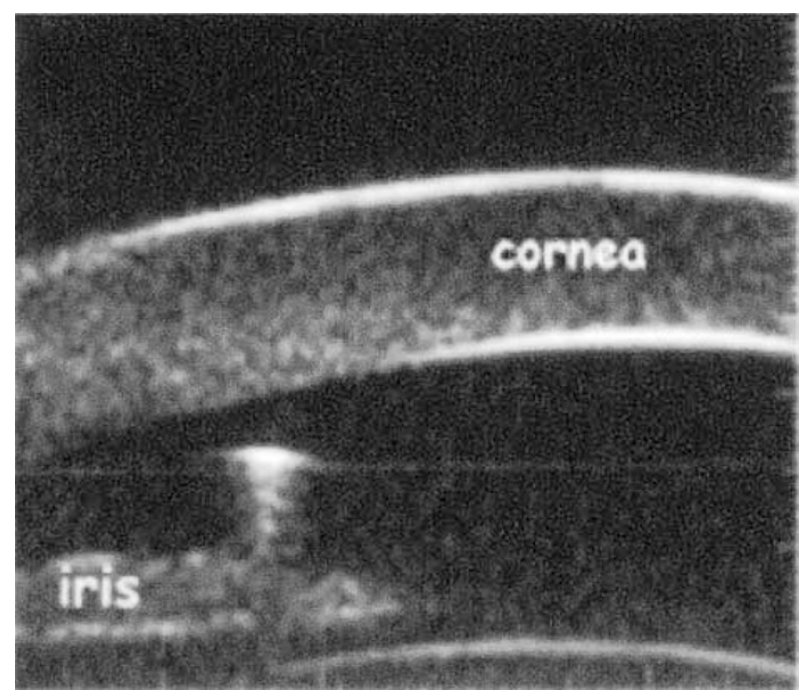

Figure 2 Intraoperative UBM (goniopuncture): cross-sectional illustration of the goniopuncture needle within the anterior chamber. There are only minimal artefacts.

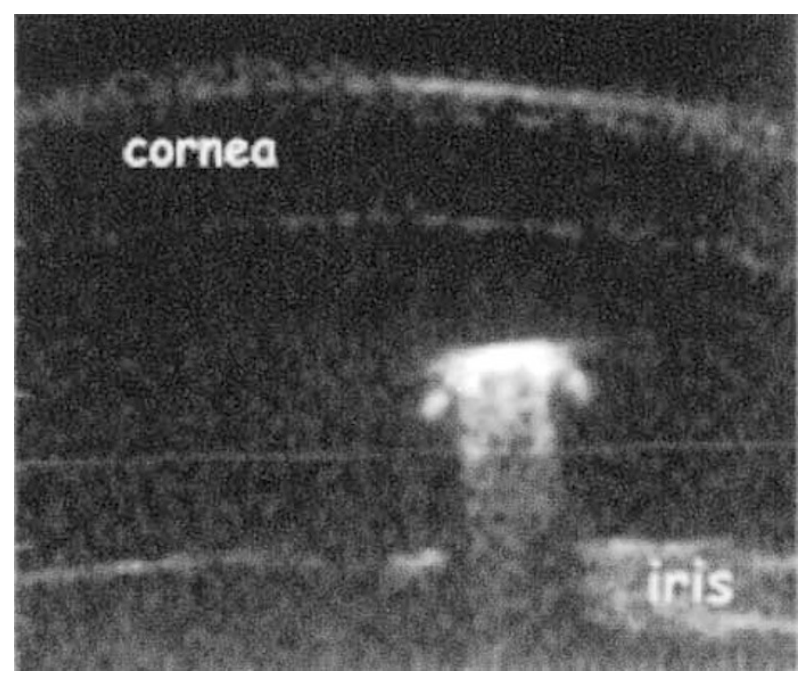

Figure 3 Intraoperative UBM (laser ablation): cross-sectional illustration of the laser endoprobe within the anterior chamber. Illustration of the underlying iris is markedly disturbed by artefacts.

bubbles (Figure 5), quantitative measurements of the ablation depths of single pulses were not feasible at all by UBM and measurements could only be made in larger ablation craters $(>150 \mu \mathrm{m})$ created by several pulses. Light microscopy after UBM-guided laser trabecular ablation generally reconfirmed the expected crater depth, inner crater diameter and the topographic localization of the laser craters in the anterior trabecular meshwork and the peripheral cornea (Table 1). There was a slight tendency in some eyes for histologically verified laser craters to be localized more anteriorly (crater-iris

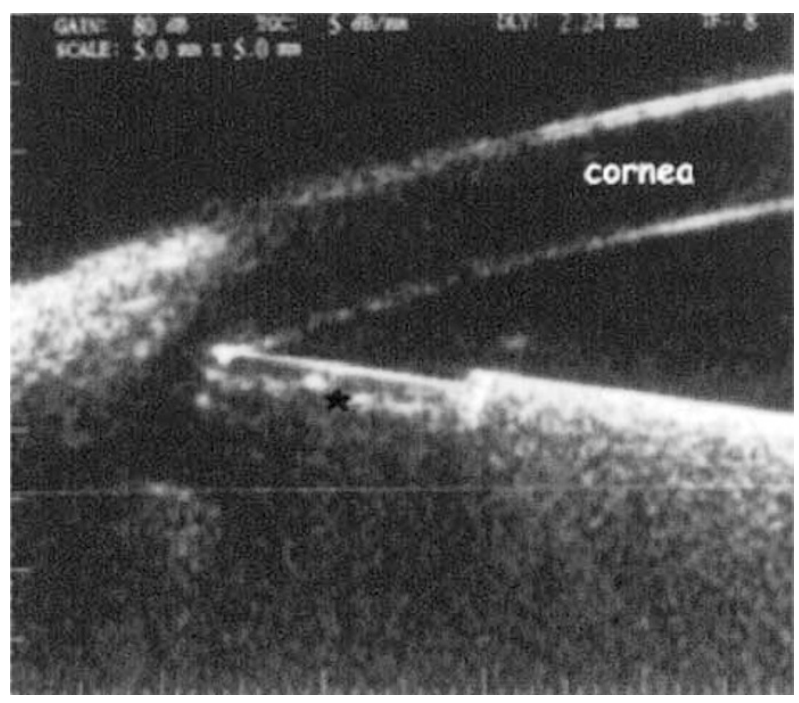

Figure 4 Intraoperative UBM (laser ablation) in a section radial to the limbus (meridional): the tip of the endoprobe (black asterisk) touches the cornea, but the area of tissue-endoprobe contact (arrow) is not sufficient for laser ablation.

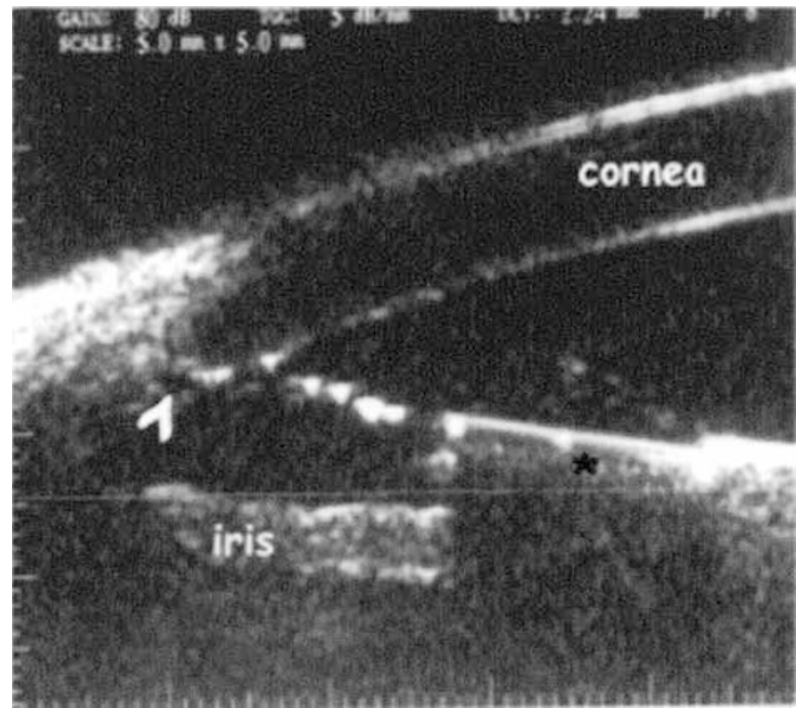

Figure 5 Intraoperative UBM (laser ablation) in a section radial to the limbus (meridional): after moving the endoprobe back the ablation area is covered with highly reflecting air bubbles. The supposed area of photovaporized tissue shows signs of hypoand hyper-reflexions (arrow). Precise quantitative measurements of the ablation depth are not possible owing to the limits of resolution.

distance, Table 1) than would have been expected from UBM guidance alone (Figure 6).

\section{Discussion}

UBM can create high-resolution images of the chamber angle region, the ciliary body, and the anterior chamber, 
regardless of corneal opacities. ${ }^{11,14}$ After cataract surgery, UBM helps to identify the topographic position of IOL haptics, ${ }^{15-17}$ especially where the anatomy of the chamber angle region is unusual, as in aniridia. ${ }^{18} \mathrm{UBM}$ is also a helpful diagnostic tool in eyes with primary or secondary dysgenetic glaucoma, in which visualization of the anterior chamber structures is often dramatically hindered by corneal opacities. ${ }^{19-21}$ UBM has also been used to assess the effectiveness of pressure-reducing mechanisms following filtration surgery since scleral drainage routes and intrascleral reservoirs can be imaged by this form of ultrasound. ${ }^{22-26}$ Ultrasound biomicroscopy of the chamber angle has to be orientated around the typical reflection patterns of specific anatomical landmarks, that is, the transition zone between sclera and cornea, ligamenta pectinata, and the ending of Descemet's membrane. ${ }^{19,20,27}$ However, the characteristic information supplied by conventional gonioscopy, such as the pigmentation of the trabecular meshwork with no specific ultrasound reflection pattern, is lost and would therefore be missed by the use of UBM alone. With a penetration depth of only $5 \mathrm{~mm}$, UBM-guided intraocular surgery cannot be used to view a larger area of the anterior chamber.

Table 1 Quantification (in $\mathrm{mm}$ ) of tissue ablation by UBM and histology $(n=4)$

\begin{tabular}{lcc}
\hline & UBM & Histology \\
\hline Crater depth & $0.37 \pm 0.06$ & $0.30 \pm 0.04$ \\
Crater diameter & $0.26 \pm 0.04$ & $0.25 \pm 0.04$ \\
Crater-iris distance & $0.46 \pm 0.16$ & $0.51 \pm 0.38$ \\
\hline
\end{tabular}

Crater depth has been slightly overestimated by UBM, possibly owing to gas bubble artefacts, while crater-iris distance has been underestimated by UBM compared to histology.
As instrument tip-tissue contact is very difficult to assess from tangential UBM pictures of the chamber angle, we did not attempt to use UBM to control the lateral motion of instruments within the anterior chamber. We therefore began by exploring the potential of UBM-guided $a b$ interno surgery for following relatively static procedures such as goniopuncture and laser trabecular ablation rather than more dynamic ones such as goniotomy and goniocurettage. To minimize image disturbance by inadvertent manual movements out of the meridional section level, a mechanical supporter was used to stabilize the position of the instruments during the procedures. The instruments could be moved slowly along the meridional axis via screw pullers. Artefactual reflections were regularly present distal to the instruments, but could be markedly reduced by moving the section level slightly to the side and attempting to hit the instruments only tangentially by UBM. There were also fewer artefact reflections with smaller instruments. With needles $<200 \mu \mathrm{m}$ in diameter, deterioration of the image through reflection from artefacts was minimal even in cross-sectional scans. In contrast to intraoperative visualization by gonioscopy or microendoscopy, UBM enables the surgeon to view the tissue in direct contact with the fibre tip during laser trabecular ablation. Owing to the chamber angle curvature, the contact between the upper edge of the endoprobe and the anterior meshwork can simulate sufficient tissue applanation when evaluated by gonioscopy, but achieves insufficient tissue-endoprobe contact for a regular tissue photovaporization when visualized under UBM (as demonstrated in Figure 6). Photoablative trabecular ablation or similar procedures can be performed more reliably under UBM guidance as the area of tissue-endoprobe contact can be controlled via the

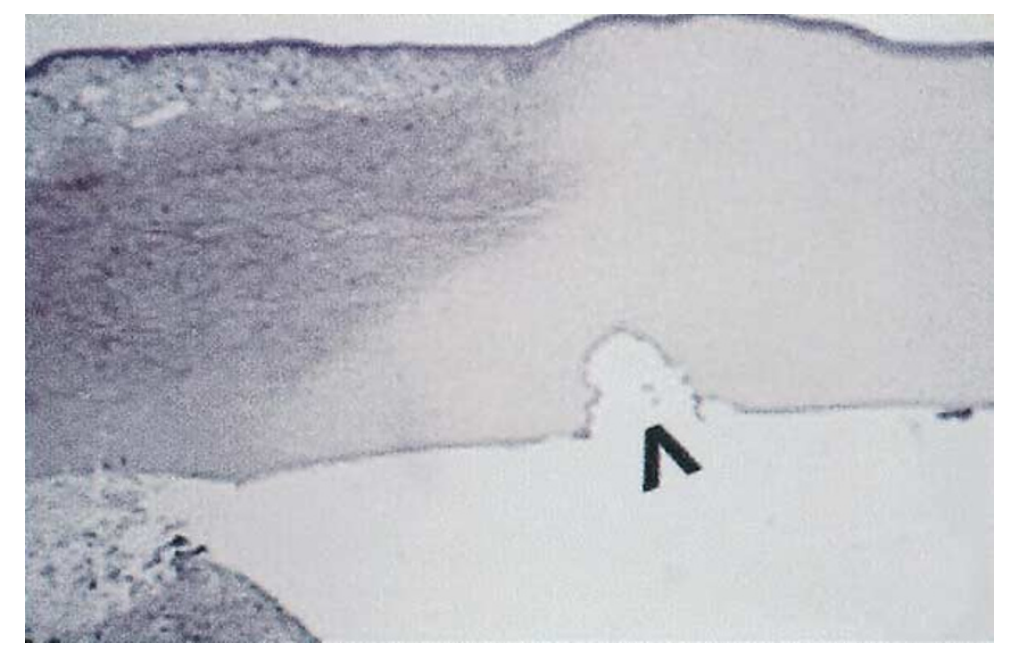

Figure 6 Light microscopy of the laser crater created by UBM-guided surgery in Figures 4 and 5. 
monitor; furthermore, the axial applanation force of the endoprobe, producing the so-called photohydraulic effect during laser emission in laser sclerostomy, ${ }^{28}$ can be better visualized by the movements of the surrounding tissue structures. From the microsurgical point of view, it would be extremely desirable to visualize the lumen of Schlemm's canal by UBM during chamber angle surgery. However, resolution limits of the $50 \mathrm{MHz}$ transducer do not allow intraoperative definition of this morphologic structure. Transducers with higher frequencies could be used for this purpose, but penetration depth would then decrease further and handling would become more difficult.

Another problem encountered in this kind of surgical technique is the difficulty of maintaining the immersion fluid within the operative field and the maintaining of surgical sterility. With our high-viscosity immersion gel, we do not need to use an eye cup to stabilize the immersion medium. However, air bubbles within the immersion gel can interfere with the quality of the ultrasound images. If the technique were to be used routinely in clinical surgery, sterility would also be a point of major concern. Sterilized immersion medium could be used as in vitreoretinal surgery. The instrument arm of the ultrasound biomicroscopy might have to be covered with a sterile coating, as in laser sclerostomy. Another practical problem could be lack of space when using instruments like the UBM and laser equipment simultaneously.

Although mechanical goniopuncture is not a standard antiglaucomatous procedure, the new era of non perforating surgery has brought new modes of application for goniopuncture. Nd:YAG laser goniopuncture has been performed by several authors following unsuccessful deep sclerectomy to open the trabeculo-descemetic membrane separating anterior chamber and intrascleral lake. ${ }^{29,30}$ Diagnostic UBM can localize the intrascleral reservoir and drainage routes, and may be of great benefit in cases where a laserassisted or a conventional surgical revision is attempted. In our opinion, UBM-guided perforation of the trabeculodescemetic membrane may even be feasible in selected cases. Although UBM-guided laser ablation with an Er:YAG laser carries the theoretical risks of any intraocular procedure (endophthalmitis, lens damage, etc), the surgical opening of the intrascleral lake or of intrascleral drainage routes can be achieved under constant visualization increasing the reliability of the intended surgical procedure.

In conclusion, UBM monitoring of limited movements of microsurgical instruments during antiglaucomatous $a b$ interno surgery such as laser trabecular ablation has the potential to supply the surgeon with additional information of value. UBM helps to illustrate the area of tissue-endoprobe contact and the penetration depth of the instrument tip within the intransparent scleral tissue while also providing a quasikinetic view of the applanation force of the instruments. If used in combination with microendoscopy or gonioscopy, UBM could well improve the intraoperative control of $a b$ interno procedures. However, several limitations of this surgical technique, such as the problems of sterility, lack of space in the surgical area and complicated and timeconsuming handling, have still to be addressed before this new surgical approach can be attempted in the clinical setting.

\section{References}

1 Barkan O. Operation for congenital glaucoma. Am J Ophthalmol 1942; 25: 552-568

2 Russell-Eggitt IM, Rice NSC, Jay B, Wyse RK. Relapse following goniotomy for congenital glaucoma due to trabecular dysgenesis. Eye 1992; 6: 197-200.

3 Russell-Eggitt IM. In defence of goniotomy (letter). $\mathrm{Br} J$ Ophthalmol 1995; 79: 709.

4 Scheie HG. Goniopuncture: an evaluation after 11 years. Arch Ophthalmol 1961; 65: 38.

5 Melamed S, Pei J, Puliafito CA, Epstein DL. Q-switched neodymium YAG laser trabeculopuncture in monkeys. Arch Ophthalmol 1985; 103: 129.

6 Hill RA, Baerveldt G, Ozler SA, Pickford M, Profeta GA, Berns MW. Laser trabecular ablation (LTA). Lasers Surg Med 1991; 11: 341-346.

7 Dietlein TS, Jacobi PC, Krieglstein GK. Erbium:YAG laser trabecular ablation (LTA) in the surgical management of glaucoma. Laser Surg Med 1998; 23: 104-110.

8 Joos KM, Alward WL, Folberg R. Experimental endoscopic goniotomy. A potential treatment for primary infantile glaucoma. Ophthalmology 1993; 100: 1066-1070.

9 Jacobi PC, Dietlein TS, Krieglstein GK. Microendoscopic trabecular surgery in glaucoma management. Ophthalmology 1999; 106: 538-544.

10 Pavlin CJ, Harasiewicz K, Sherar MD, Foster FD. Clinical use of ultrasound biomicroscopy. Ophthalmology 1991; 98: 287-295.

11 Pavlin CJ, Harasiewicz K, Foster FD. Ultrasound biomicroscopy of anterior segment structures in normal and glaucomatous eyes. Am J Ophthalmol 1992; 113: 381-389.

12 Spaeth GL, Azuara-Blanco A, Araujo SV, Augsburger JJ. Intraobserver and interobserver agreement in evaluating the anterior chamber angle configuration by ultrasound biomicroscopy. J Glaucoma 1997; 6: 13-17.

13 Tello C, Liebmann J, Potash SD, Cohen H, Rich R. Measurement of ultrasound biomicroscopy images: intraobserver and interobserver reliability. Invest Ophthalmol Vis Sci 1994; 35: 3549-3552.

14 Azuara-Blanco A, Spaeth GL, Araujo SV, Augsburger JJ, Katz LJ, Calhoun JH et al. Ultrasound biomicroscopy in infantile glaucoma. Ophthalmology 1997; 104: 1116-1119.

15 Pavlin CJ, Harasiewicz K, Foster K. Ultrasound biomicroscopic analysis of haptic position in late-onset, recurrent hyphema after posterior chamber lens implantation. J Cataract Refract Surg 1994; 20: 182-185. 
16 Pavlin CJ, Rootman D, Arshinoff S, Harasiewicz K, Foster K Determination of haptic position of transsclerally fixated posterior chamber intraocular lenses by ultrasound biomicroscopy. J Cataract Refract Surg 1993; 19: 573-577.

17 Steiner A, Steinhorst UH, Steiner M, Theischen M, Winter R. Ultrasound biomicroscopy (UBM) for the localization of artificial lens haptics after transscleral suture fixation. Ophthalmology 1997; 94: 41-44.

18 Schweykart N, Reinhard T, Engelhardt S, Sundmacher R. Postoperative ultrasound biomicroscopic evaluation of the tangible position of black diaphragm posterior chamber lenses in congenital and traumatic aniridia in comparison with gonioscopy. Klin Monatsbl Augenheilkd 1999; 214: 381-390.

19 Stegman Z, Sokol J, Liebmann JM, Cohen H, Tello C, Ritch R. Reduced trabecular meshwork height in juvenile primary open-angle glaucoma. Arch Ophthalmol 1996; 114: 660-663.

20 Dietlein TS, Engels BF, Jacobi PC, Krieglstein GK. Ultrasound biomicroscopic patterns after glaucoma surgery in congenital glaucoma. Ophthalmology 2000; 107: 1200-1205.

21 Engels B, Dietlein TS, Jacobi PC, Krieglstein GK. Ultrasound biomicroscopic diagnosis of congenital glaucoma. Klin Monatsbl Augenheilkd 1999; 215: 338-341.

22 McWhae JA, Crichton ACS. The use of ultrasound biomicroscopy following trabeculectomy. Can J Ophthalmol 1996; 31: 187-191.

23 Chiou AGY, Mermoud A, Hediguer SEA, Schnyder CC, Faggioni R. Ultrasound biomicroscopy of eyes undergoing deep sclerectomy with collagen implant. $\mathrm{Br} J$ Ophthalmol 1996; 80: 541-544.

24 Chiou AGY, Mermoud A, Underdahl JP, Schnyder CC. An ultrasound biomicroscopic study of eyes after deep sclerectomy with collagen implant. Ophthalmology 1998; 105: 746-750.

25 Avitabile T, Uva MG, Russo V, Ott JP, Paulick B, Reibaldi A. Beurteilung des Filterkissens mittels Ultraschallbiomikroskopie. Klin Monatsbl Augenheilkd 1998; 212: 101-105.

26 Yamamoto T, Sakuma T, Kitazawa Y. An ultrasound biomicroscopic study of filtering blebs after mitomycin C trabeculectomy. Ophthalmology 1995; 102: 1770-1776.

27 Klemm M, Bergmann U, Guthoff R. Die Ultraschallbiomikroskopie als Kriterium der Funktionsprüfung-des suprachoroidalen Spalts nach kammerwinkelchirurgischen Eingriffen. Klin Monatsbl Augenheilkd 1997; 210: 74-77.

28 Fankhauser F, Durr U, England C, Kwasniewska S, Van der Zypen E, Henchoz PD. Optical principles related to optimizing sclerostomy procedures. Ophthalmic Surg 1992; 23: 752-761.

29 Karlen ME, Sanchez E, Schnyder CC, Sickenberg M, Mermoud A. Deep sclerectomy with collagen implant: medium term results. Br J Ophthalmol 1999; 83: 6-11.

30 Mermoud A, Karlen ME, Schnyder CC, Sickenberg M, Chiou AG, Hediguer SE et al. Nd:YAG goniopuncture after deep sclerectomy with collagen implant. Ophthalmic Surg Lasers 1999; 30: 120-125. 